\title{
Black Holes and Entropy: A Skeptical Perspective
}

\author{
Ben Akih-Kumgeh
}

Thermodynamics and Combustion Laboratory, Department of Mechanical and Aerospace Engineering, Syracuse University, Syracuse, NY 13244, USA; bakihkum@syr.edu

\begin{abstract}
Black holes are objects of significant interest in modern cosmology. From what initially looked like a superficial analogy between black hole mechanics and thermodynamics, a new epistemological framework has emerged according to which far-reaching conclusions about black hole can be reached through thermodynamic analysis. An example of this is the view that the temperature of a black hole is inversely proportional to its mass. This paper raises doubts about the currently accepted connection between black holes and entropy. It does so by first reviewing the principles of thermodynamics and the properties a system must have in order to admit of proper thermodynamic analysis. It is argued that the current view of black holes preclude their distinct classification either as closed or open systems, a fact which has a bearing on the formulation of the First and Second Laws. From a mechanistic view of temperature and heat, combined with my recent work on the physical meaning of classical entropy, I show that the generalized Second Law of black hole thermodynamics is probably in error. The notion of heat transfer (which is central to entropy definition) is not explicit in the black hole energy equation. To address the challenges raised, black hole mechanics must either commit to a phenomenological approach and therefore only invoke thermodynamics in the classical sense or accept a microscopic view of black hole matter in order to readily draw from established results of statistical mechanics. It is argued that a proper connection to classical thermodynamics would lead to the view that the temperature of a black hole increases with its mass, as a result of which a positive specific heat capacity is to be expected, contrary to the prevailing doctrine.
\end{abstract}

Keywords: black holes; entropy; generalized second law; black hole temperature

\section{Introduction}

Black holes are objects of ongoing scientific research. They also appeal to our sense of wonder about the universe and when we loosely combine them with entropy, an often misunderstood and liberally extrapolated physical concept, we obtain an aesthetically appealing but possibly incorrect blend of science and science-fiction. In this paper, I will try to show that our prevailing understanding of black hole properties demands extra care in any attempts to connect them to thermodynamics. It seems that this has not been the case in developing the notions of black hole entropy and black hole temperature, making it possible that we may be in error.

I will first review the principles of thermodynamics, especially the classification of thermodynamic systems and the corresponding First and Second Law formulations. Entropy in classical and statistical thermodynamics is briefly reviewed, in connection to my recent work on an alternative interpretation of classical entropy. The review of thermodynamics is intended to help us identify what properties a physical system should have in order to be properly analyzed using thermodynamic concepts.

In this work, I will not consider entropy and information to be synonymous. It seems reasonable to recognize two views of entropy in physical science: (1) The phenomenological approach in which we stay close to Clausius' definition of the term using heat and temperature. Along this line may be added my recent suggestion to construe entropy change as a non-dimensional measure of changes 
The 3rd International Electronic and Flipped Conference on Entropy and Applications (ECEA 2016), 1-10 November 2016; Sciforum Electronic Conference Series, Vol. 3, 2016

in a system's energy upon interaction with another system/universe. (2) The statistico-mechanical approach in which we connect entropy to counting mechanical objects with given mechanical properties (momenta and spatial locations). Information, being neither a classical thermodynamic nor mechanical quantity, then appears to be an extraneous property introduced into physical science for no epistemic virtue other than further confusion. Although there is a sense in which one may make an analogy between counting mechanical objects with momenta and positions in statistical thermodynamics and counting permutations of symbols used in communication, such counting cannot be perceived as leading to a conserved quantity, as sometimes suggested.

A brief review of central concepts in black hole cosmology is then presented, distinguishing between views about black holes before and after general relativity theory. Since the notion of black holes and entropy surfaces in Berkenstein [1] and Bardeen et al. [2], I try to sketch the reasoning that led to the connection. I modestly set out to show that it is reasonable to be skeptical of this connection, especially as it is further made to imply that black hole temperatures increase as the mass decreases (negative specific heat capacity). I speculate, albeit without a serious commitment, that a proper connection of black holes to thermodynamics would lead to the notion that black temperatures increase with increasing mass, contrary to the prevailing position.

\section{Relevant Background}

\subsection{Fundamentals of Thermodynamics}

The science of thermodynamics maybe understood to be a framework to investigate the behavior of a carved out region of the universe containing matter as this region interacts with the rest of the universe through exchange of energy and possibly mass. To apply this framework to the investigation of objects, such as black holes, a review of key concepts and principles is needed.

\subsubsection{Systems and Properties}

The object of thermodynamic investigation is the system. By a system we understand a carved out region of the universe containing matter with known properties at a given state. Thermodynamics deals with average properties, so that there are no spatial variations within the system for which well defined system properties are given. Since exchange of mass and energy is important, simplification of system analysis is carried out by classifying systems on the basis of possible energy and mass exchanges.

An isolated system is one in which neither mass nor energy can be exchanged with the rest of the universe. If we do not admit of spatial variations within a well defined stable system, then such a thermodynamic system might appear to be boring. The isolated system becomes interesting if we admit temporal variations of its properties (resulting from initial heterogeneity in its properties).

A closed system is one in which exchange of energy with the environment is possible but mass can neither enter nor leave the system. We can therefore investigate the response of the system to energy exchange processes. For this, knowledge of the energy exchange process and material response properties is needed. One broadly distinguishes between energy transfer on account of temperature differences and energy transfer through mechanical deformations of the system boundaries, the driving forces for these deformations being of various types other than temperature gradients.

An open system can exchange energy and mass with the environment. We may then investigate how such a system responds to exchange of mass, energy, or both with the environment. These distinctions lead to differences in the forms of the associated expressions of the First and Second Laws of thermodynamics. By extension, such differences would mean that transfer of thermodynamic analysis to black hole mechanics simply by analogies is not warranted unless a justified commitment to one type of system is made. 
The 3rd International Electronic and Flipped Conference on Entropy and Applications (ECEA 2016), 1-10 November 2016; Sciforum Electronic Conference Series, Vol. 3, 2016

The equivalence of energy and mass in relativity through $M c^{2}=E$ removes the distinction between closed and open systems. We can then only reasonably speak of isolated and closed/open systems in relativity. If mass transfer occurs through radiative energy transfer (photon masses), we may approximately treat the problem as a closed system.

For a closed system, the principle of energy conservation or the First Law simply states that the energy of the system increases on account of heat or work addition. In engineering terms, one prefers to say that the energy of the system can increase as a result of heat addition or it can decrease as a result of work expenditure. The First Law takes the form:

$$
d E=\delta Q+\sum_{i=1}^{N} \delta W_{i}=\delta Q+\sum_{i=1}^{N} F_{i} d X_{i}
$$

where $Q$ is heat transferred into the system, $F_{i}$ a generalized force and $X_{i}$ is the associated generalized coordinate which undergoes a differential change around an equilibrium state.

Significant progress in thermodynamics came from adopting an underlying model of matter in the macroscopic systems. It has proven to be very useful to interpret observed macroscopic variables in terms of the mechanics of constituent particles since Thomson, Maxwell, and Clausius. If this picture is true, then the non-mechanical notions of heat and temperature can be eliminated from fundamental laws of thermodynamics. Our adherence to these concepts is purely a matter of linguistic and cultural inertia.

- The boundaries of a thermodynamic system divides the universe into two: within is the system being investigated and without is the rest of the universe.

- The First Law therefore only states that the energy of the system within increases $\left(d E_{S}\right)$ by the same amount as the energy of the rest of the universe decreases $\left(d E_{E}\right)$.

- We may divide the different ways in which the rest of the universe energetically interacts with the system into two groups: energy exchange by virtue of differences in temperature $d E_{E, T}$ and non-thermal interactions broadly classed as work, $d E_{E, W}$. The First Law becomes:

$$
d E_{S}=d E_{E, T}+d E_{E, W}=d E_{E, T}+\sum_{i=1}^{N} F_{i} d X_{i}
$$

If we view all energy terms involved to be reducible to the kinetic and potential energy of the constituent particles (or their statistical averages), we then have a First Law of Thermodynamics independent of the notion of heat (i.e. we made the substitution $\delta Q=d E_{E, T}$-differential heat is simply change in the environmental internal energy through a microscopic energy transfer mechanism - conduction, convection or radiation). This can be useful in deciding which terms in the energy equation should be associated with the entropy of a black hole that interacts with another part of the universe such as a second black hole. Clausius' definition of entropy targets thermal non-equilibrium i.e. $\delta Q, d E_{E, T}$ or $d E_{S, T}$.

A few comments about the notion of generalized forces and coordinates. The science of thermodynamics took shape in the 19th century after the success of analytical mechanics. The notion of potentials was transferred from mechanics to the new field initially by Massieu [3]. This was followed by further developments in the hands of Helmholtz, Duhem [4], and Gibbs [5]. One views the generalized force, $F_{i}$, as describing the response behavior of the systems energy to changes in the associated coordinate i.e. $F_{i}=\frac{\partial E_{S}}{\partial X_{i}}$. 
The 3rd International Electronic and Flipped Conference on Entropy and Applications (ECEA 2016), 1-10 November 2016; Sciforum Electronic Conference Series, Vol. 3, 2016

\subsubsection{Second Law and Entropy}

We shall only consider the Second Law and its connection to entropy for a closed system. As discussed in my previous work [6], Clausius' definition of entropy through its differential

$$
d s=\frac{\delta q}{T}
$$

can be made to reflect a non-dimensional measure of energy changes as a system interacts with another or the environment through heat transfer. The modified form suggested was

$$
d s=\frac{\delta q}{k T}=\frac{\delta q}{\epsilon}
$$

with the further emphasis that the Second Law is the law of heat transfer. For a constant volume heat transfer process without any other driving forces, we find upon integrating that $s_{2}-s_{1}=\ln \left(\epsilon_{2} / \epsilon_{1}\right)$. In the unmodified form, this has the associated heat capacity and is expressed in terms of ratios of temperatures $s_{2}-s_{1}=c_{v} \ln \left(T_{2} / T_{1}\right)$. Clausius' definition allows for the substitution $\delta Q=T d S$ in Equation (1) to get:

$$
d E=T d S+\sum_{i=1}^{N} F_{i} d X_{i}
$$

This is now the most widely used fundamental thermodynamic potential due to Gibbs but the close connection of entropy to heat transfer should not be forgotten. That is, as presented here the only driving force leading to entropy change is temperature difference. If one is interested in capturing the potential for a certain relaxation process in nature, one may well define a new kind of entropy if terms of the driving force, i.e., $d S_{X i}=\frac{F_{i} d X_{i}}{T}$. Historically, there is one instance in which this approach was taken and it led to what is considered to be entropy production in chemically reacting systems. This approach is due to De Donder [7], and later taken up by his student, Prigogine in developing the fluctuation-dissipation theory and chaos in chemical reactors [8]. In this view, the driving forces are the different chemical potentials of the species $\left(\mu_{i}=\frac{\partial E}{\partial N_{i}}\right)$

$$
d S_{i}=\sum \frac{\mu_{i} d N_{i}}{T}
$$

The entropy change due to heat transfer is then indicated as $d S_{e}$. This makes the thermodynamic potential

$$
d E=T d S_{e}+T d S_{i}+\sum_{i=1}^{N} F_{i} d X_{i}
$$

where $F_{i}$ now represents driving forces other than temperature and chemical potential differences. Macroscopically considered, the First Law therefore requires us to bear in mind the origin of the entropy terms. In most cases, this is heat transfer but in chemical reactors, this may include entropy production due to reconfiguration of the chemical associations among atoms. This reconfiguration can further make available potential energy in the form of kinetic or thermal energy without heat crossing the system boundary.

In statistical thermodynamics, the focus shifts from average system properties to counting of states and probabilities. Boltzmann entropy change for a microcanonical ensemble is a measure of the change in the number of ways:

$$
s_{2}-s_{1}=k \int_{1}^{2} \frac{d W}{W}=k \ln \frac{W_{2}}{W_{1}}
$$


The 3rd International Electronic and Flipped Conference on Entropy and Applications (ECEA 2016), 1-10 November 2016; Sciforum Electronic Conference Series, Vol. 3, 2016

where $W$ is the multiplicity or the number of ways the energy of the system can be distributed among its available energy states. In the non-dimensional fashion in my previous work, this would amount to $s_{2}-s_{1}=\ln \frac{W_{2}}{W_{1}}$.

Gibbs entropy at a given state, on the other hand, is closer related to Boltzmann's H-theorem and entropy in information theory.

$$
s=-k \sum_{i=1}^{W} \rho_{i} \ln \rho_{i}
$$

If one considers a system with $W$ states and equal probabilities, then $\rho_{i}=1 / W$ and we end up with statistical entropy differences as in Equation (8). These views of entropy therefore require that we either focus on phenomenological processes and use Clausius' or De Donder's entropy definitions or commit to a microscopic and statistical view of the investigated system.

\subsection{Black Hole Mechanics}

The basic notions of black hole mechanics can be understood to have ties to both classical and relativistic mechanics. As is to be expected, some notions have been transferred from the understanding of black holes within a classical framework to that of general theory of relativity. The very name of these physical objects is committed to a classical mechanical legacy as discussed below.

\subsubsection{Black Holes before the General Theory of Relativity}

Two central ideas precede the development of black holes in classical mechanics. The first is Newton's theory of gravity, according to which two masses, $M$ and $m$, at a given separation, $r$, experience a mutual attractive force, $T$, quantitatively determined as:

$$
F=\frac{G M m}{r^{2}}
$$

The potential energy, $P$, of the mass, $m$, at a given separation from the other mass, $M$, can be found to be

$$
P=-\frac{\partial F}{\partial r}=-\frac{G M m}{r}
$$

According to this relation the potential energy almost vanishes at infinite separations and grows negatively unbound as $r$ approaches zero. The mass $m$ moving with a velocity, $v$, also has kinetic energy, $K$.

One can then introduce the notion of escape velocity. It is imagined that one would have to project the mass $m$ from near the surface of the more massive object $M$, with a certain minimum velocity, $v_{e}$, so that as the kinetic energy diminishes with increasing separation, a point is reached where near vanishing of the kinetic energy coincides with near vanishing of the potential energy. Imposing the principle of conservation of energy of the mass $m$ means that at all times during the flight, $K+P=0$ or $1 / 2 m v^{2}-G M m / r=0$. At the point of projection where the separation is $R_{0}$, we have $v_{e}=\sqrt{2 G M / R_{0}}$. That is, the escape velocity is uniquely determined by the mass of the "planet", $M$, and the separation at the point of projection. Although the mass of the moving object, $m$, does not feature in this result, the arguments leading to the equation are presented in terms of Newtonian gravity as well as potential and kinetic energies.

One could simply ask: given $M$ and a "true" particle projected with the speed of light, $c$, what should the radius of projection, $R_{0}$, be for the particle to escape the gravitational field of the "planet"? This radius of project is near the surface of $M$. At this point one simply uses the speed of light as a stand-in for the velocity of any mass that interacts with $M$ in accordance with Newtonian gravity and possesses kinetic and potential energies in the classical sense. If one knows by experience that no object can travel with a speed higher than $c$, the question therefore sets a limit to projecting mass-like gravitationally constrained particles from the surface of the planet. 
The 3rd International Electronic and Flipped Conference on Entropy and Applications (ECEA 2016), 1-10 November 2016; Sciforum Electronic Conference Series, Vol. 3, 2016

The natural philosopher, the Reverend John Michell in 1784 [9] went beyond these considerations with two key assumptions: (1) Newton's gravity holds true and (2) light is made of corpuscle and by extension, light corpuscles are endowed with mass, interact with massive objects $M$ according to Newton's gravitational law and possess both kinetic and potential energies which can be calculated as above. From these consideration, he concluded that every massive object has a certain radius such that if its mass is compressed to that radius, projecting a corpuscle of light with the maximum allowable with a velocity, $c$, the corpuscle will fail to escape the planet and eventually fall back to the surface. This assumes non-interaction with any other heavenly bodies. The massive object, $M$, thus configured is a black hole, lost to observers outside a certain radius.

A similar idea was developed by Laplace in his Exposition du systeme du Monde [10]. However, it seems that he was led by increasing experimental evidence of the wave nature of light to omit the treatment in later editions of his book. It seems then that the initial fascination with black holes waned in the 19th century coinciding with the less popular corpuscular theory of light.

With this change of the ontological status of light we may only retain the notion of "black hole" and consider Michell's investigation to have established that "an object capable of gravitational interaction with another massive object, if its velocity is not to exceed the speed of light, must be projected with this limiting velocity from a well defined radius. At separations less than this critical radius, the gravitational object projected with the limiting speed of light executes projectile motion and returns to the surface of that massive planet.

\subsubsection{Black Holes after the General Theory of Relativity}

From the 20th century onward, black holes are interpreted as consequences of certain solutions of Einstein's field equation of the general relativity theory (GTR). The GTR relates the curvature of space-time manifold with the stress-energy tensor of a gravitational field. Central to the theory is differential geometry. In special relativity the Lorentian metric of the space-time manifold is given as

$$
d s^{2}=d t^{2}-d x^{2}-d y^{2}-d z^{2}
$$

In GTR, geometry varies from point to point, captured by the metric tensor, $g_{a b}$, so that a line element can described by

$$
d s^{2}=g_{a b} d x_{a} d x_{b}=g_{00} d t^{2}+g_{i j} d x_{i} d x_{j} \text { with }(i, j=1,2,3)
$$

The gravitational field can be captured by specifying space-time using the metric $g_{a b}$. It is not the intention of this paper to go into the field equations. Solutions of these field equations for specific cases have been obtained. One of these was the Schwarzschild solution for a stationary spherical mass whose metric is given as

$$
d s^{2}=-\left(1-\frac{2 M}{r}\right) d t^{2}+\left(1-\frac{2 M}{r}\right)^{-1} d r^{2}+r^{2} d \Omega^{2}
$$

This solution leads to a singularity at $r=2 M$ ( or $r=2 G M / c^{2}$ in physical units of distance), very much similar to the critical radius of Michell's black hole. It is known that initial fascination with these singularities and their connection to the 18th century notion of black holes was not enthusiastically embraced by Einstein and Eddington, another leading expert in GTR [11].

Much later, other solutions of the field equations emerged. One of these is the Reissner-Nordstrom (RN) solution which considers a spherically symmetric black hole of mass, $M$, and electric charge, $Q$. The $R N$ metric can be written as

$$
d s^{2}=-\left(1-\frac{2 M}{r}+\frac{Q^{2}}{r^{2}}\right) d t^{2}+\left(1-\frac{2 M}{r}+\frac{Q^{2}}{r^{2}}\right)^{-1} d r^{2}+r^{2} d \Omega^{2}
$$


The 3rd International Electronic and Flipped Conference on Entropy and Applications (ECEA 2016), 1-10 November 2016; Sciforum Electronic Conference Series, Vol. 3, 2016

From solution of the field equations only the electromagnetic field emerges as the only non-zero component $F=Q / r^{2}$. It also features singularities at $r=2 M$.

A very important solution of the field equations is that due to Kerr. It deals with a black hole of mass, $M$, rotating with an angular momentum, $J$. One form of the Kerr metric is

$$
d s^{2}=-\left(1-\frac{2 M r}{\rho}\right) d t^{2}-\frac{4 M \operatorname{arsin}^{2} \theta}{\rho^{2}} d t d \phi+\frac{\Sigma}{\rho^{2}} \sin ^{2} \theta d \theta^{2}+\frac{\rho^{2}}{\Delta} d r^{2}+\rho^{2} d \theta^{2}
$$

The mass of a Kerr black hole has been shown by Smarr [12] to be related to the surface area, angular momentum, and charge. In modern texts this can be written as

$$
M=\frac{\kappa A}{4 \pi}+2 \Omega J+\phi Q
$$

where the curvature the black hole is $\kappa=\frac{1}{4 M}$.

\section{Connection between Black Holes and Thermodynamics}

The connection between black holes and thermodynamics starts with introducing the notion of black hole entropy through the first law.

\subsection{Current Views on Black Hole Entropy}

The first explicit connection between black holes and entropy is due to Berkenstein [1]. The abstract of his 1973 paper opens with: "There are a number of similarities between black hole physics and thermodynamics. Most striking is the similarity in the black hole area and of entropy: Both quantities tend to increase irreversibly."

Since the principle of entropy increase is understood to mean that (1) the net entropy of two systems interacting by heat transfer increases or (2) the entropy of a system to which heat is added increases, the irreversible increase of the entropy of a black hole can only be considered to be generally true if black holes only receive energy but never loses any energy through heat transfer processes, such as radiation.

The First Law of black hole mechanics can be derived from the differential of mass in the Smarr formula, treating curvature, angular velocity, and electromagnetic potential as driving forces and varying the surface area, angular momentum, and charge. A second path, more rigorous is the one presented by Bardeen et al. [2] in which the interaction of two black holes is considered. This black hole mass differential is

$$
d M=\frac{\kappa}{8 \pi G} d A+\Omega d J+\phi d Q
$$

Berkenstein considers Equation (18) to be analogous to the fundamental thermodynamic potential, $d E=T d S-p d V$, in which pressure is the only force by which mechanical work can be added or withdrawn from the system. It is argued that the entropy of a black hole, $S$, is related to area, $S=f(A)$ on the basis of the similarity. What is missed is that the more basic form of the thermodynamic potential is related to elemental heat exchange:

$$
d E=T d S-p d V \Longleftrightarrow d E=\delta Q-p d V
$$

The analogy is further pursued $[1,13]$ to establish that the entropy of a black hole is

$$
S=\frac{c^{3}}{4 G \hbar} A
$$

where $\hbar$ is the reduced Planck constant. The temperature is found to be

$$
T=\frac{\hbar}{2 \pi k c} \kappa
$$


The 3rd International Electronic and Flipped Conference on Entropy and Applications (ECEA 2016), 1-10 November 2016; Sciforum Electronic Conference Series, Vol. 3, 2016

It should be noted that this connection is only based on analogy. A distinction between the mechanisms of energy exchange between a black hole and the universe is not offered nor is a clear connection to entropy definitions previously discussed.

\subsection{Criticism and Alternative Connection between Black Hole and Entropy}

The main skepticism about validity of the above thermodynamic connection centers on the use of analogy to justify the connection between the black hole surface area and entropy. Since this is the main foundation of black hole thermodynamics, if the analogy falls apart, then the series of deductions that follow from it, such as black hole temperature are also in question. It is argued here that

- $T d S \neq \kappa d A$. The surface area has no connection to heat transfer or statistics of constituent microscopic particles. The surface gravity, $\kappa$, is just another non-thermal driving force conjugate with the surface as its generalized coordinate.

- If $T d S \neq \kappa d A$, then there is no justification for $T=\kappa \approx \frac{1}{4 M}$

- If $T \neq \frac{1}{4 M}$, then the heat capacity $C=\frac{\partial M}{\partial T}$ is not necessarily negative. It might or might not be.

How might we correctly identify an analog of thermodynamic entropy in black hole mechanics? There are two ways to invoke entropy in the analysis of systems such as black holes. One is to appeal to the classical approach in which, for closed systems, entropy is defined in terms of heat transfer. The second approach would require us to apply statistical mechanical reasoning in the more physically illuminating approach of Boltzmann and Maxwell. Regarding the first approach, we must identify which of the terms in the Smarr formula or the two black hole mass transfer model would count as heat.

Let us consider the interaction of two black holes that leads to mass accretion by one. A two step-approach is needed to account for the change of mass/energy and how that change of mass/energy leads to readjustment of the generalized coordinates of the black hole.

Consider the case of two Kerr black holes interacting by mass/energy exchange. If one first treat them to proceed so fast, such that the surface area, angular momentum and charge remain fairly constant, we may proceed to analyze how entropy changes, defined in terms of mass/energy exchange, can be determined. We consider two masses, $M_{1}$ and $M_{2}$. Through mass accretion, the first receives $\delta M_{1}$ and the mass/energy conservation now requires that

$$
\delta M_{1}+\delta M_{2}=0
$$

Since $\delta M_{1}>0, M_{2}$ loses mass as a result of the interaction. Let's define entropy changes in the non-dimensional manner proposed in previous work [6]. We seek to discover the relation between the two masses for the resulting entropy to be greater than zero in accordance with the Second Law.

$$
d s_{1}=\frac{\delta M_{1}}{M_{1}} \text { and } d s_{2}=\frac{\delta M_{2}}{M_{2}}=-\frac{\delta M_{1}}{M_{2}}
$$

If we consider both masses to be sufficiently large as to remain fairly constant during the mass exchange process, then we can establish the total entropy change to be

$$
d s_{1}+d s_{2}=\frac{\delta M_{1}\left(M_{2}-M_{1}\right)}{M_{1} M_{2}}
$$

That is, for the total entropy change to be positive, it must be the case that $M_{2}>M_{1}$. We must qualify that this is only directly related to entropy if the accretion proceeds through radiative heat transfer, and not through mechanical mass transfer. For each of the black holes, we now have the difference between initial and final entropies as $s_{f}-s_{i}=\ln \frac{M_{f}}{M_{i}}$. Further, here we have our first suggestion that if the notion of temperature must be introduced it would be such that 
The 3rd International Electronic and Flipped Conference on Entropy and Applications (ECEA 2016), 1-10 November 2016; Sciforum Electronic Conference Series, Vol. 3, 2016

$$
M c^{2}=N_{\text {photons }} h v=N_{B H \text { particles }} k T
$$

where we have introduced the speed of light to obtain energy units for the mass. The mechanical theory of heat links the temperature of a gas to the average kinetic energy of its particles. The average velocity of ideal gas particles is comparable with the speed of sound, so that $T \propto a^{2}$. In seeking the temperature of a black hole, one must have in mind the ontology of black holes as consisting of some fundamental particles. Given that we pay closer attention to the relativistic energy, considering black holes as consisting of gas particles might not be very helpful. We do not have any knowledge of the speed of sound and what state of matter black holes have. I argue that we can draw from the analogy of temperature of ideal gases as being proportional to the speed of sound, the elementary particles being those capable of translational motion. In the case of black holes, we can anchor our speculation on photons and their frequencies

$$
\frac{M}{N_{\text {photons }}} \frac{c^{2}}{k}=\frac{h}{k} v=T
$$

We note that $h, k$, and $c$ are physical constants. It follows then that the temperature of a black hole may be related to the average frequency (or wave number) of its photons which together make up the energy of the black hole or it could be related to the average mass of a representative photon, $M / N$, seeing that $c$ is a constant i.e. $T \propto M$, and not $T \propto M^{-1}$. If this is true it also implies that the heat capacity, $\frac{\partial M}{\partial T}$, is greater than zero, unlike the negative behavior resulting from the current temperature definition. It should be further noted that non-thermal mass exchange does not permit definition of entropy change as carried out above.

We may proceed to consider what happens after the rapid mass loss/accretion in each of the two black holes. This corresponds to the mechanical relaxation of a thermodynamic system that has been subjected to non-equilibrium state, almost instantaneously. Here one might simply invoke the variational form of the Smarr formula and recognize that the mass differential can be related to entropy according to Equation (23).

$$
d M=\frac{\kappa}{8 \pi G} d A+\Omega d J+\phi d Q=M d s
$$

In this form, $\kappa, \Omega$, and $\phi$ are generalized non-thermal (electro or mechanical) forces with $A, J$, and $Q$ the corresponding generalized coordinates, similar to the fundamental thermodynamic potential. There is no reason to prefer to connect the surface area to entropy over the angular momentum or the charge. In terms of analogy in classical thermodynamics, the term associated with surface gravity and surface area would be comparable with surface tension, not temperature.

A few questions remain, especially given that this author is not writing from a cosmological perspective. Can a black hole lose energy through radiation? What is the exact principle forbidding this? How does the temperature change upon mass accretion or upon heat transfer? It would appear that if temperature is related to the average photon frequency, then heat loss by radiation from the black hole should lead to a lower average photon frequency, hence lower temperature. If photons are not created or destroyed in the transfer process, then loss of energy by radiation should lead to a decrease of mass of the black hole. We noted that of the two interacting black holes, the entropy change would be such that one experiences an entropy decrease while the mass receiving the accreted mass element would increase. Having related these masses to the temperature and average photon frequency through $M c^{2}=N h v=N k T$, it can be concluded that two black holes interacting by mass/energy exchange would do so naturally is the one losing energy has a higher average photon frequency than the one receiving. 
The 3rd International Electronic and Flipped Conference on Entropy and Applications (ECEA 2016), 1-10 November 2016; Sciforum Electronic Conference Series, Vol. 3, 2016

\section{Conclusions}

In its current state, the connection between black holes and entropy is not satisfactory. This paper examines the basics of thermodynamics and considers whether these align with the First Law of black hole mechanics. It is argued that the analogy on the basis of which entropy is introduced into black hole mechanics is not satisfactory. If this is the case, the definition of temperature also suffers. A plausible alternative is considered according to which the entropy change is a non-dimensional change of black hole mass resulting from a mass/energy exchange. Temperature would appear to be proportional to the mass of a black hole, leading to a normal positive specific heat capacity.

Acknowledgments: Support is acknowledged from the Syracuse University College of Engineering and Computer Science and the Syracuse Center of Excellence for Environmental and Energy Systems.

Author Contributions: The research problem is conceived, carried out, and reported by the author with appropriate acknowledgment.

Conflicts of Interest: The author declares no conflict of interest.

\section{References}

1. Bekenstein, J.D. Black holes and entropy. Phys. Rev. D 1973, 7, 2333.

2. Bardeen, J.M.; Carter, B.; Hawking, S.W. The four laws of black hole mechanics. Commun. Math. Phys. 1973, 31, 161-170.

3. Massieu, F. Thermodynamique: Mêmoire sur les fonctions catactéristiques des divers fluides et sur la théorie des vapeurs; Académie des Sciences de L'Institut National de France: Paris, France, 1876; volume 22. (In French)

4. Duhem, P.M.M. Le potentiel thermodynamique et ses applications à la mécanique chimique et à l'étude des phénomènes électriques. Deuxième tirage; A. Hermann: Paris, France, 1895. (In French)

5. Gibbs, J.W. On the equilibrium of heterogeneous substances. Am. J. Sci. 1878, 16, 441-458.

6. Akih-Kumgeh, B. Toward Improved Understanding of the Physical Meaning of Entropy in Classical Thermodynamics. Entropy 2016, 18, 270.

7. De Donder, T.; Van Rysselberghe, P. Thermodynamic Theory of Affinity; Stanford University Press: Palo Alto, CA, USA , 1936; volume 1.

8. Kondepudi, D.; Prigogine, I. Modern Thermodynamics: From Heat Engines to Dissipative Structures; John Wiley \& Sons: Hoboken, NJ, USA , 2014.

9. Michell, J. On the Means of Discovering the Distance, Magnitude, \&c. of the Fixed Stars, in Consequence of the Diminution of the Velocity of Their Light, in Case Such a Diminution Should be Found to Take Place in any of Them, and Such Other Data Should be Procured from Observations, as Would be Farther Necessary for That Purpose. By the Rev. John Michell, B. D. F. R. S. In a Letter to Henry Cavendish, Esq. F. R. S. and A. S. Phil. Trans. R. Soc. Lond. 1784, 74, 35-57.

10. Laplace, P.S. Exposition du système du monde; Bachelier: Paris, France, 1835. (In French)

11. Thorne, K. Black Holes E Time Warps: Einstein's Outrageous Legacy (Commonwealth Fund Book Program); WW Norton \& Company: New York, NY, USA, 1995.

12. Smarr, L. Mass formula for Kerr black holes. Phys. Rev. Lett. 1973, 30, 71.

13. Poisson, E. A Relativist's Toolkit: The Mathematics of Black-Hole Mechanics; Cambridge University Press: Cambridge, UK, 2004.

(C) 2016 by the authors; licensee MDPI, Basel, Switzerland. This article is an open access article distributed under the terms and conditions of the Creative Commons Attribution (CC-BY) license (http://creativecommons.org/licenses/by/4.0/). 